\title{
Heterostructures for Realizing Magnon-Induced Spin Transfer Torque
}

\author{
P. B. Jayathilaka, ${ }^{1}$ M. C. Monti, ${ }^{2}$ J. T. Markert, ${ }^{2}$ and Casey W. Miller ${ }^{1}$ \\ ${ }^{1}$ Department of Physics, University of South Florida, 4202 East Fowler Avenue, Tampa, FL 33620, USA \\ ${ }^{2}$ Department of Physics, University of Texas at Austin, 1 University Station C1600, Austin, TX 78712, USA
}

Correspondence should be addressed to Casey W. Miller, millercw@usf.edu

Received 17 February 2012; Accepted 21 April 2012

Academic Editor: Giancarlo Consolo

Copyright ( 2012 P. B. Jayathilaka et al. This is an open access article distributed under the Creative Commons Attribution License, which permits unrestricted use, distribution, and reproduction in any medium, provided the original work is properly cited.

\begin{abstract}
This work reports efforts fabricating heterostructures of different materials relevant for the realization of magnon-induced spin transfer torques. We find the growth of high-quality magnetite on $\mathrm{MgO}$ substrates to be straightforward, while using transition metal buffer layers of $\mathrm{Fe}, \mathrm{Cr}, \mathrm{Mo}$, and $\mathrm{Nb}$ can alter the structural and magnetic properties of the magnetite. Additionally, we successfully fabricated and characterized $\mathrm{Py} / \mathrm{Cr} / \mathrm{Fe}_{3} \mathrm{O}_{4}$ and $\mathrm{Fe}_{3} \mathrm{O}_{4} / \mathrm{Cr} / \mathrm{Fe}_{3} \mathrm{O}_{4}$ spin valve structures. For both, we observe a relatively small giant magnetoresistance and confirm an inverse dependence on spacer layer thickness. Thus, we have shown certain materials combinations that may form the heterostructures that are the building blocks necessary to achieve magnon-induced spin transfer torque devices.
\end{abstract}

\section{Introduction}

The prediction $[1,2]$ and observation of "spin transfer" or "spin-transfer torque" in mechanical point contacts [3], and subsequently in lithographically defined point contacts [4], and nanopillars [5], revealed that spin-polarized currents can manipulate magnetization dynamics. The interest in this area is dominated by the potential for spin-torque-based magnetic random access memory to become a universal memory [6], as well as interesting high-frequency devices based on spin torque oscillators (STOs) $[7,8]$. Spin torque research is one of the key areas of spintronics and nanomagnetism today [9], being relevant to both science and technology.

Theoreticians have recently promoted the idea of initiating spin transfer torque by thermal transport from magnons, initially in all-metal systems $[11,12]$ and recently with insulators [10]. Slonczewski's concept in [10] is based on the ability to convert the spin momentum of magnons in a magnetic insulator into a polarized current in a normal metal, which is then driven by a thermal gradient into a metallic ferromagnet. He showed that the quantum yield of heat-driven in-plane spin-transfer torque can exceed that from electric current in state-of-the-art spin-transfer devices utilizing magnetic tunnel junctions by nearly two orders of magnitude. STOs would benefit greatly from the increased quantum yield deliverable from this magnon-induced spin transfer torque (MISTT) because increasing the usable torque would increase their output power, which is not yet compatible with applications [13-15]. Spin torque-based devices in general would benefit from replacing the highcurrent densities now necessary for operation, as this causes appreciable heating, as well as vortex nucleation in the free layer via the unavoidable Oersted field. MISTT could address both of these issues, allowing significant improvements in device performance, fabrication requirements, and reliability. The experimental realization of this magnonic spin torque may thus have transformative impact by essentially creating a new class of spin torque devices.

The significant difference between existing spin-transfer devices and Slonczewski's proposal is the origin of the torque on the free layer. Traditionally, the spin-transfer torque comes from direct electrical injection of electrons from a fixed metallic ferromagnet through a normal metal into the free layer [9]. The spin polarization of these electrons mimics that of the fixed ferromagnet, which results in an imposed torque on the free layer magnetization when the 


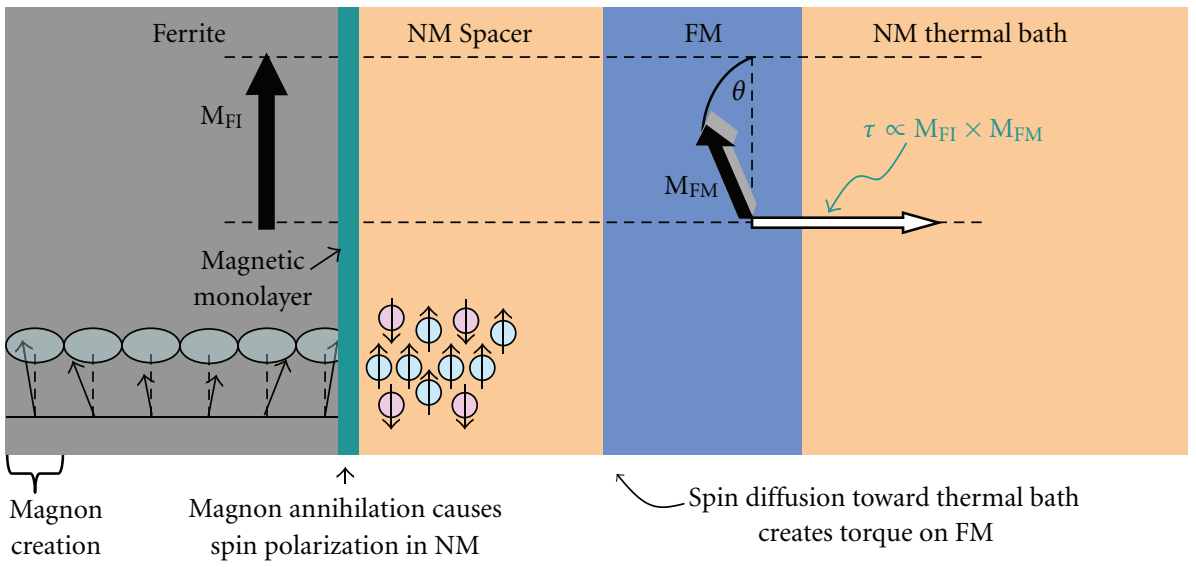

FIGURe 1: Magnon-induced spin transfer torque (MISTT) ala Slonczewski [10]. Magnons are created thermally in the magnetic insulator. Though these magnons are annihilated at the ferrite-normal metal (NM) interface (which contains a monolayer of ferromagnet metal atoms), their spin momentum is transferred to the electrons in the NM spacer. This creates spin accumulation in the NM, which then diffuses toward the NM thermal bath. The spin accumulation current density (more precisely, its time derivative) at the Py interface imposes a torque on the Py magnetization if its magnetization $\mathrm{M}_{\mathrm{FM}}$ is at some angle relative to the ferrite's magnetization, $\mathrm{M}_{\mathrm{FI}}$.

spin polarization axes of the two ferromagnets are not colinear. In the MISTT device concept (shown schematically in Figure 1), spin polarized electrons still impose a torque on the free layer via a normal metal spacer, but there are several critical differences. First, the electrons are driven by a thermal—not electrical—gradient. Second, because the fixed layer in MISTT is a resistive ferri or ferromagnet (such as spinel ferrites), electrons cannot be simply injected, so a more exotic mechanism for polarizing the electrons in the normal metal is required: magnon annihilation. The magnons within the ferrite carry spin momentum, and, being bosons, the magnon population can be increased dramatically through heating. Conversely, these magnons can also be annihilated when they reach the ferrite-normal metal interface. The spin momentum originally carried by the annihilated magnons can be transferred to electrons in the normal metal, causing them to become spin polarized. These spin-polarized electrons then diffuse into the free magnet, leading to a spin-transfer torque.

Given their great potential, we have been exploring the materials combinations that may lead to the successful fabrication of these devices. This article outlines our progress toward the production of structures that may be useful for realizing magnonic spin transfer torque devices.

\section{Experimental Section}

All the films were grown in an Ar ion sputtering system with a base pressure of $20 \mathrm{nTorr}$. Ultra-high-purity gases were used for all the deposition. Samples were rotated at a speed of 40 rotations per minute during the deposition to obtain uniform film thickness. To grow different thicknesses of the same material at a single deposition, the rotation of the sample holder was stopped, so that one sample closer to the gun sees more material flux and grows thicker and the samples away from the target get less material flux and grows thinner. This was used to grow "wedges" for thickness-dependent studies. In this study, three different sets of samples were grown. Those are (a) $\mathrm{Fe}_{3} \mathrm{O}_{4}$ on $\mathrm{MgO}$ (100) substrates with transition metal buffer layers ( $\mathrm{Fe}$, $\mathrm{Cr}, \mathrm{Nb}, \mathrm{Mo}$ and No buffer), (b) $\mathrm{Py} / \mathrm{Cr} / \mathrm{Fe}_{3} \mathrm{O}_{4}$ spin valve with different $\mathrm{Cr}$ thicknesses on $\mathrm{Si}$ (100) substrates, and (c) $\mathrm{Fe}_{3} \mathrm{O}_{4} / \mathrm{Cr} / \mathrm{Fe}_{3} \mathrm{O}_{4}$ substrates with different $\mathrm{Cr}$ thicknesses on $\mathrm{MgO}(100)$ substrates. $\mathrm{Fe}_{3} \mathrm{O}_{4}$ was grown at $300 \mathrm{C}$ by rf reactive sputtering from an $\mathrm{Fe}$ target in an $\mathrm{Ar}$ and $\mathrm{O}_{2}$ environment. The amount of $\mathrm{Ar}$ and $\mathrm{O}_{2}$ were controlled by the adjusting the flow rates of those gases in to the deposition chamber (20 SCCM Ar and 0.75 SCCM O $\mathrm{O}_{2}$ ). The total deposition pressure was $10 \mathrm{mTorr}$ and the deposition rate was $0.26 \AA / \mathrm{S}$. Epitaxy was confirmed by X-ray diffraction, and the quality of the $\mathrm{Fe}_{3} \mathrm{O}_{4}$ was indicated by the quality of the Verwey transition. The $\mathrm{Fe}_{3} \mathrm{O}_{4}$ films we grew showed the Verwey transition in between $119 \mathrm{~K}$ to $125 \mathrm{~K}$, which suggests the stoichiometry was $\mathrm{Fe}_{3} \mathrm{O}_{4-x}$, with $x$ bounded above by $0.001[16]$.

\section{High-Quality Ferrite Films on MgO Substrates}

Since magnetite and $\mathrm{MgO}$ have nearly perfect lattice matching (as do most spinel ferrites), it is hard to distinguish them using the principal reflections in XRD because the intensity of the substrate (002) peak drowns out the magnetite's (004) peak. We have used a combination of wide angle XRD to observe the (004) and (008) peaks of the substrate and film [17], along with in-plane XRD (i.e., phi scans) to distinguish the $\mathrm{Fe}_{3} \mathrm{O}_{4}$ from the substrate peaks. We carried out scans focusing on the (311) and (220) orientations. Figures 2(a) and 2(d) show the results of these scans, which confirm the epitaxial growth of the magnetite on the $\mathrm{MgO}$. Using higher resolution in Figures 2(b), 2(c), 2(e), and 2(f) shows that the magnetite peaks are broadened slightly relative to those of the substrate, which may be expected from finite-size effects. The full width at half maximum (FWHM) for $\mathrm{MgO}$ (311), 


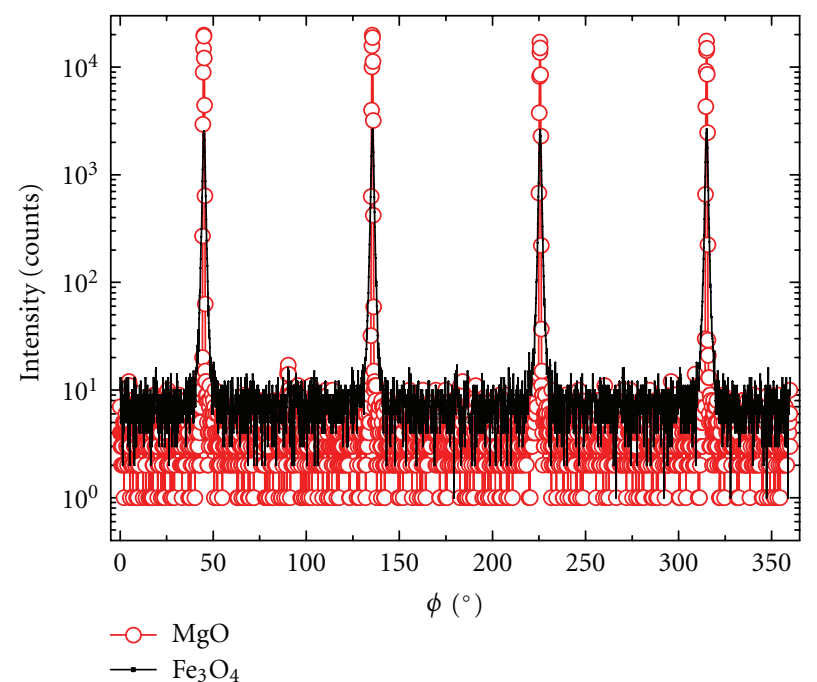

(a)

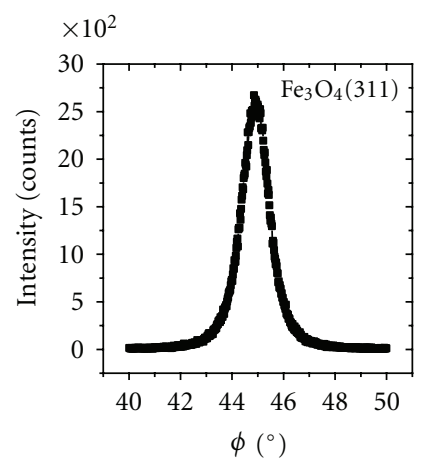

(b)

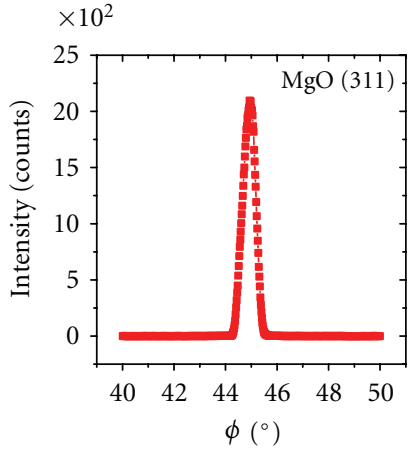

(c)

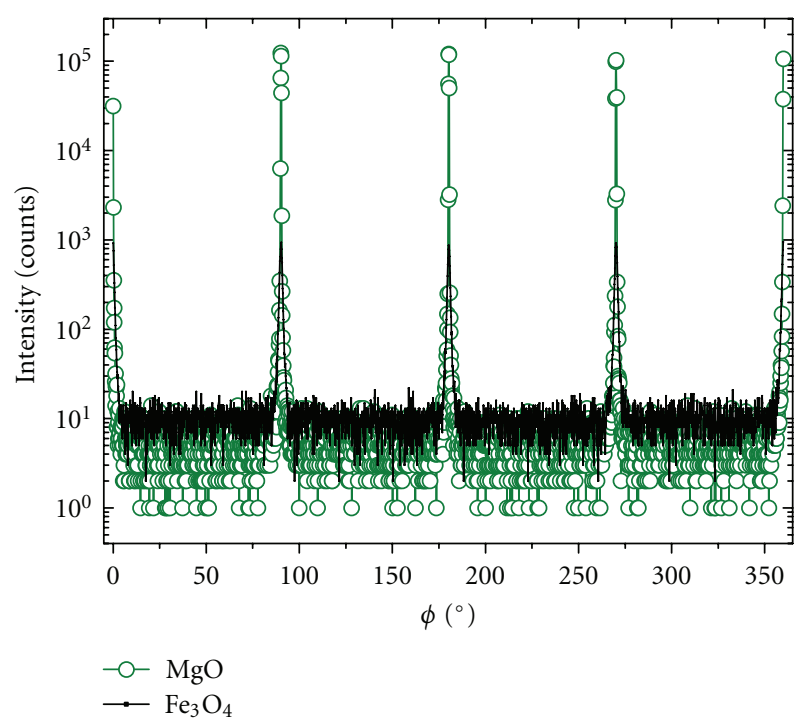

(d)

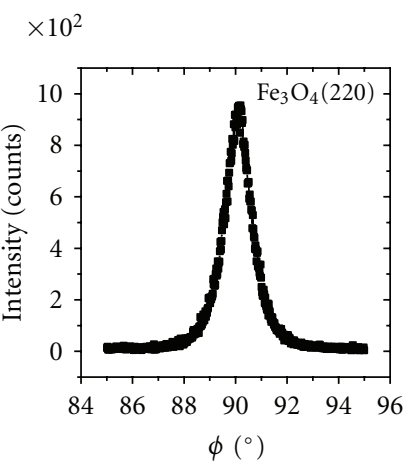

(e)

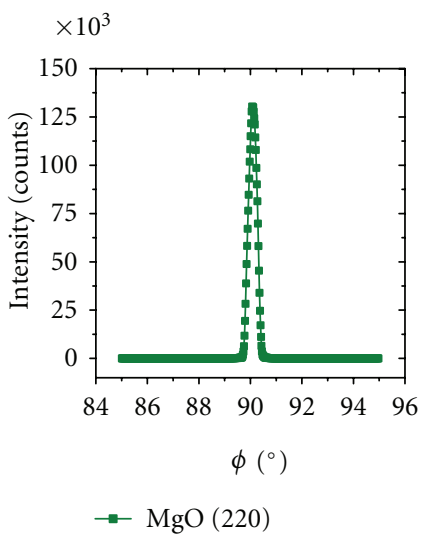

(f)

Figure 2: XRD Phi scans of $\mathrm{Fe}_{3} \mathrm{O}_{4}$ on $\mathrm{MgO}$ (100) substrates. (a) Complete scan on (220) plane, (b) complete scan on (311) plane, (c) MgO (220) peak, $\mathrm{FWHM}=0.38^{\circ}$, (d) $\mathrm{Fe}_{3} \mathrm{O}_{4}(220)$ Peak, $\mathrm{FWHM}=1.36^{\circ}$ (e) $\mathrm{MgO}$ (311) peak, $\mathrm{FWHM}=0.56^{\circ}$ (f) $\mathrm{Fe}_{3} \mathrm{O}_{4}(311)$ peak, $\mathrm{FWHM}=$ $1.34^{\circ}$.

$\mathrm{Fe}_{3} \mathrm{O}_{4}$ (311), $\mathrm{MgO}$ (220), and $\mathrm{Fe}_{3} \mathrm{O}_{4}$ (220) was $0.56^{\circ}, 1.34^{\circ}$, $0.38^{\circ}$, and $1.36^{\circ}$ respectively.

\section{Impact of Thin Buffer Layers on Magnetite}

In a previous study, we showed that, the magnetite can be grown on $30 \AA$ buffer layers of $\mathrm{Fe}, \mathrm{Mo}, \mathrm{Nb}$, and $\mathrm{Cr}$ [17]. $\mathrm{Fe}, \mathrm{Mo}$, and $\mathrm{Nb}$ lend themselves to varying degrees of (100) texture, with $\mathrm{Nb}$ being the lowest quality; Cr leads to polycrystalline magnetite. The Mo-buffered magnetite has lattice parameters similar to magnetite grown directly on $\mathrm{MgO}$, which is somewhat strained relative to bulk magnetite. The large lattice mismatch between Mo and magnetite appears to lead to the growth of magnetite nanoparticles, not a film per se. The Fe-buffered sample shows the least amount of strain of any sample. This reduced strain appears to reduce the density of antiphase boundaries, as inferred from mobile magnetic domains observed with temperaturedependent MFM. This is in contrast to magnetite grown directly on $\mathrm{MgO}$ [18].

Resistivity measurements were carried out to determine the effect of the transition metal buffer layers on the Verwey transition, as shown in Figure 3. In growing, the magnetite on these buffer layers an in situ mask exchange system allowed us to grow the transition metals on separate $\mathrm{MgO}$ (100) substrates individually, and then to grow the magnetite simultaneously on all the samples in one deposition run (i.e., preparation took place entirely under vacuum). Since the magnetite is the same on all the samples, any differences in resistivities should be due to the buffer layer. In measuring the resistivity, we attached the samples to a measurement probe and dip the end into a liquid nitrogen bath, allowing the sample temperature to cool from $300 \mathrm{~K}$ down to about $100 \mathrm{~K}$ in about 30 minutes. Because of the 


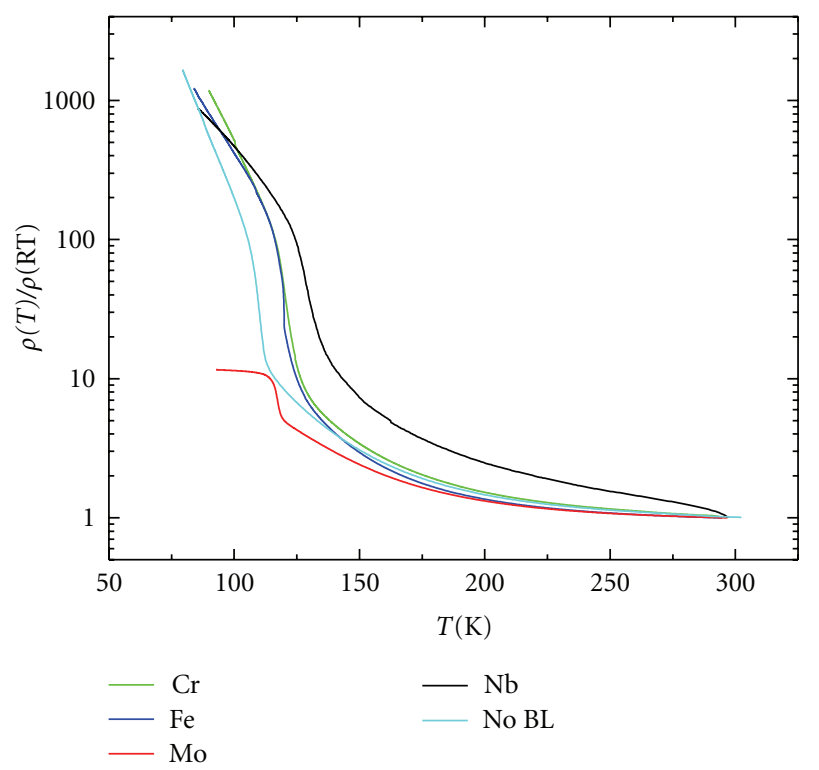

FIGURE 3: Change in resistivity as a function of temperature for magnetite simultaneously grown on different transition metal buffer layers, relative to the room temperature resistivity.

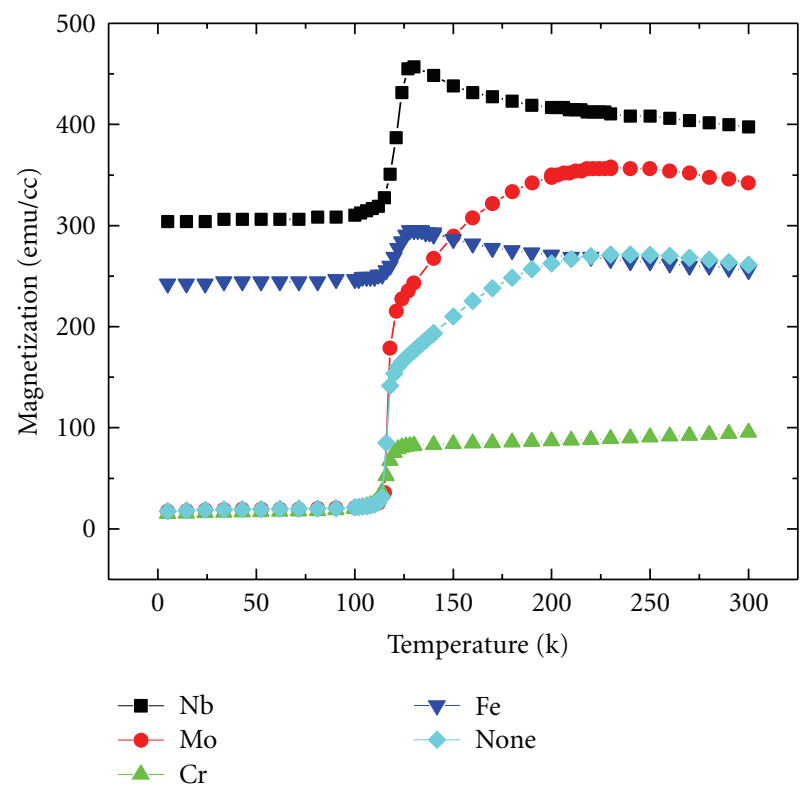

Figure 4: Temperature dependent magnetization of the $\mathrm{Fe}_{3} \mathrm{O}_{4}$ grown on top of different buffer layers. These SQUID magnetometry measurements were performed with a measurement field of 100 Oe applied along the (110) direction of the $\mathrm{MgO}$.

high resistivity, we used 2 point resistivity measurements, making contact to the sample with pressed indium. The resistivity is measured as the sample cools down; the cooling rate is sufficiently slow that no thermal lag is noted with subsequent warming.

The unbuffered magnetite shows the sharpest Verwey transition, with an increase in resistivity of more than an order of magnitude in just a few degrees. $\mathrm{Cr}, \mathrm{Fe}$, and $\mathrm{Nb}$ buffered samples showed little higher transition temperatures than the unbuffered magnetite, but the transition was not sharp. This shift in the transition temperature may be due to the stress created from these buffer layers on the magnetite layer, or possibly the formation of an interfacial layer between the buffer and the magnetite (e.g., if the buffer became oxidized by reducing the magnetite, then the interfacial magnetite may have its stoichiometry altered). The Mo-buffered sample showed a transition point closer to the unbuffered sample, but the resistance saturates, unlike any other samples we investigated. We hypothesize this being due to the formation of conduction paths at grain boundaries; indeed, force microscopy images show that the Mo-buffered sample's topography is quite granular due to the formation of magnetite nanoparticles rather than a film. Additionally, these grains appear to have magnetic switching events during magnetic force microscopy that are caused by sample-tip interactions. This indicates the formation of grains that are weakly coupled to their neighbors.

The Verwey transition can also be observed through magnetization measurements. Figure 4 shows the magnetization as a function of temperature with a 100 Oe applied field along the (110) direction (along the diagonal of the substrate plane) for each of the samples. We see each sample has a distinct drop in magnetization at the Verwey transition, which is the typical behavior. It is notable that the $\mathrm{Fe}$ and $\mathrm{Nb}$-buffered samples' behavior is distinct from measurements made with the field along the (100) direction on the same samples. We previously observed anomalous magnetization peaks in these samples along (100) direction at the Verwey transition [17]. Thus, it seems that the magnetite anisotropy is temperature dependent, leading to a temperature-dependent rotation of the easy axis above and below the Verwey transition; this manifests itself as a peaking magnetization if the moment rotates into the direction sampled by the SQUID. Similar behavior was reported in bulk Magnetite [19]. Measurement with a vector magnetometer should clarify these observations.

Figure 5 shows hysteresis loops of buffered and unbuffered magnetite samples along the (100) direction at temperatures above $(150 \mathrm{~K})$ and below $(10 \mathrm{~K})$ the Verwey transition. The samples with no buffer, Mo, and Cr buffers all have similar behavior, showing enhanced coercive and saturation fields at low temperatures. There is also a shearing of the low-temperature loops. On the other hand, the Feand $\mathrm{Nb}$-buffered samples show an increasing coercivity at low fields, but the loop shape is not as dramatically changed. This could be due to these buffer layers being more prone to oxidation than the other buffer materials used, which may cause the interfacial magnetite to be reduced.

\section{Magnetite-Based Spin Valves}

The magnetite was introduced into spin valve structures. The $\mathrm{Cr}$ spacer layers were grown as a wedge to study the thickness dependence. Current in-plane (CIP) magnetoresistance measurements were carried out at room temperature for $\mathrm{Py} / \mathrm{Cr} / \mathrm{Fe}_{3} \mathrm{O}_{4}$ and $\mathrm{Fe}_{3} \mathrm{O}_{4} / \mathrm{Cr} / \mathrm{Fe}_{3} \mathrm{O}_{4}$ spin valve structures. 


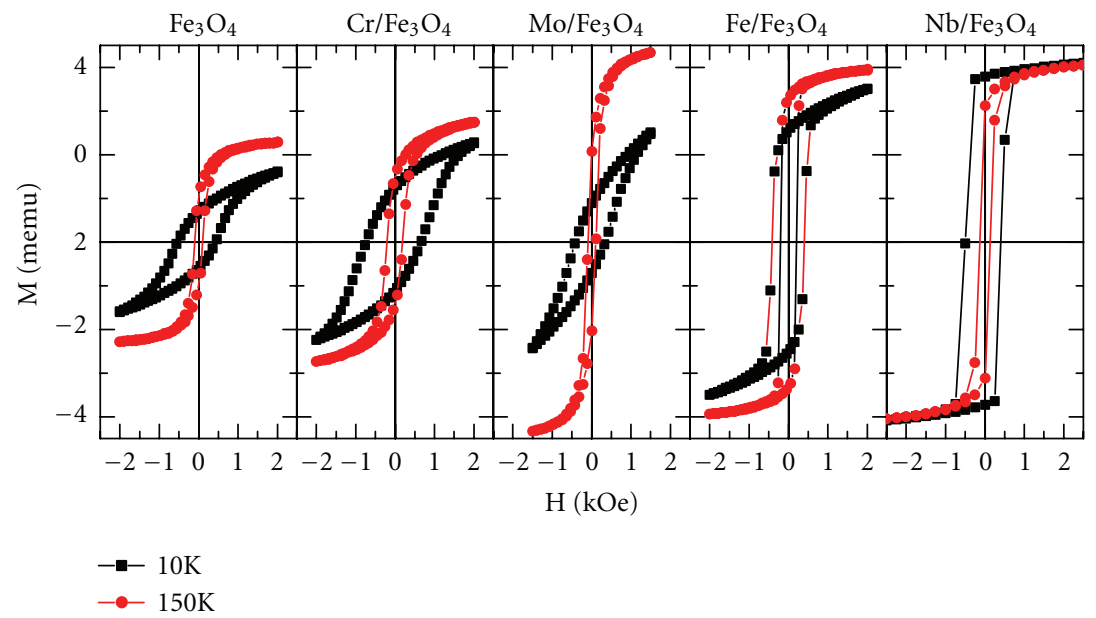

FIGURE 5: Hysteresis loops for $\mathrm{Fe}_{3} \mathrm{O}_{4}$ grown simultaneously on $\mathrm{MgO}$ (100) substrates with and without $30 \AA$ transition metal buffer layers. The SQUID magnetometry measurements were performed with a measurement field of 100 Oe applied along the (100) direction of the $\mathrm{MgO}$.

While CIP measurements are not typically used in spintorque devices, this method can indicate that spin-dependent scattering is present in the structure. Note that MISTT does not even need current to flow in the device, as the magnons may be generated thermally (e.g., by optical means). We used pseudo-four-point resistance measurements, in which $\mathrm{I}^{+}$and $\mathrm{V}^{+}$leads are connected together at a single Indium contact (similarly for $\mathrm{I}^{-}$and $\mathrm{V}^{-}$) in order to remove effects related to the leads. In $\mathrm{Py} / \mathrm{Cr} / \mathrm{Fe}_{3} \mathrm{O}_{4}$ spin valves, the $\mathrm{Cr}$ thickness was varied from $41 \AA$ to $107 \AA$ and the $\mathrm{Py}$ and $\mathrm{Fe}_{3} \mathrm{O}_{4}$ thicknesses were kept constant at $300 \AA$ (Figure 6). The GMR was calculated by subtracting the resistance of the saturated parallel state by the resistance of the shoulder in the antiparallel state. Then, the percentage was calculated with respect to the resistance of the saturated parallel state. The magnetoresistance showed a decrease with increasing spacer layer thickness in $\mathrm{Py} / \mathrm{Cr} / \mathrm{Fe}_{3} \mathrm{O}_{4}$. A fit of this thickness dependence reveals a decay constant of about $19 \AA$. This is less than the $45 \AA$ reported for the spin diffusion length of $\mathrm{Cr}$ at $4.2 \mathrm{~K}$ [20]. Since the thicknesses used for the $\mathrm{Cr}$ spacer layer were relatively large, we did not observe any oscillatory coupling behavior as a function of spacer layer thickness. This ensured we were able to switch both ferromagnets separately, and it indicates the existence of a GMR signal, even for large thicknesses of normal metal spacer layers. In addition to the GMR signal there is also an anisotropic magnetoresistance (AMR) peak of Py due to the reversal of magnetization by the applied field. This AMR signal is essentially unavoidable because the metallic Py and Cr have much lower resistivity than the magnetite, so the current flows primarily through these layers. The intensity of this peak and its field range can be reduced by applying a magnetic field during the growth process, and likely by lithographic patterning to take advantage of shape anisotropy [21].

In $\mathrm{Fe}_{3} \mathrm{O}_{4} / \mathrm{Cr} / \mathrm{Fe}_{3} \mathrm{O}_{4}$ spin valves (Figure 7 ), the $\mathrm{Cr}$ thickness was varied from $57 \AA$ to $151 \AA$. To get different coercive fields from the magnetite, the bottom layer was $500 \AA$ and the top layer was $1000 \AA$. Here, there are no well-defined parallel and antiparallel states due to the butterfly-like AMR signal intrinsic to the magnetite layers [22]. Thus, we calculated the GMR by taking the shift of the signal relative to the AMR signal. In this case, the magnetoresistance signal also decays exponentially with the spacer layer thickness, but the decay constant is $33 \AA$. We did not observe any oscillatory coupling due to higher spacer layer thickness.

The GMR values are quite small for both spin valve varieties relative to all-metallic CIP spin valves [23], but fall in the range of GMR reports for magnetite-normal metalferromagnetic systems $[22,24]$. This is most likely related to a combination of factors, which most likely includes possible oxidation of the $\mathrm{Cr}$ at the magnetite interface acting as a barrier to spin-dependent reflections necessary for large GMR, as well as the conductivity mismatch between the magnetite and the metals shunting much of the current in the Py-containing devices. The differences in the two decay constants observed in the two spin valves is probably related to impurities in the spacer (which may be due to residual oxygen from the reactive sputtering to form the magnetite), which are known to impact the spin diffusion length [20], and possibly uncertainty in the spacer layer thickness.

\section{Conclusions}

We have studied the growth of magnetite on transition metal buffer layers and the formation of magnetite-based spin valves. We found the structural and magnetic properties of magnetite to be strongly dependent on the buffer layer. Despite the differences noted for Cr buffer layers, we were able to grow spin valves that use $\mathrm{Cr}$ as a spacer layer and magnetite as the top layer. While these spin valves do not appear to have promise for GMR applications, this work shows we have successfully fabricated heterostructures with 


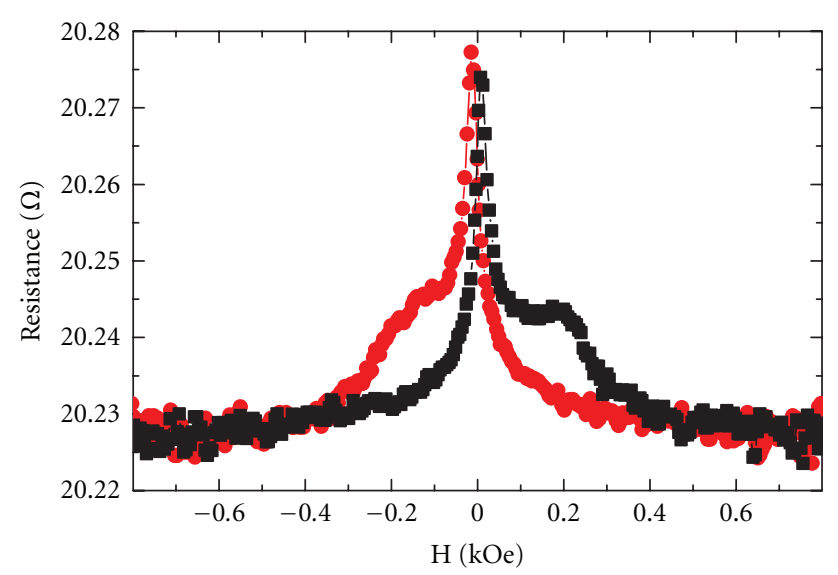

(a)

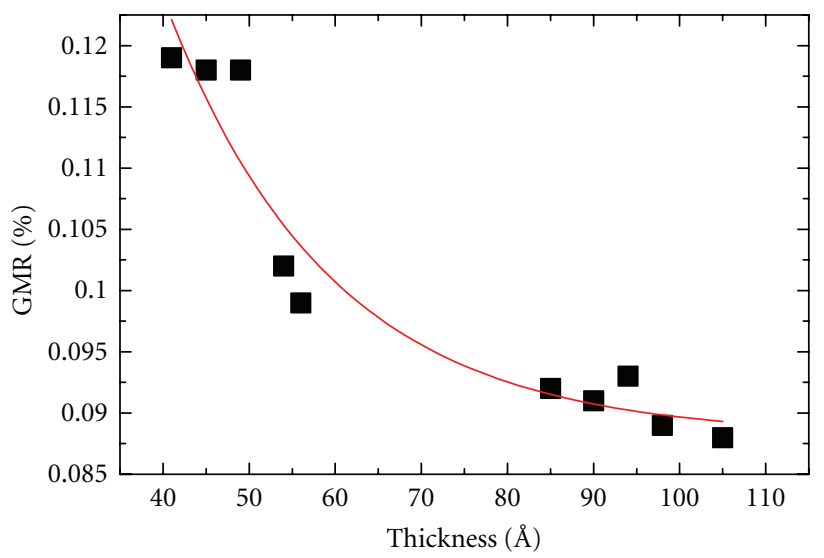

(b)

Figure 6: (a) Room temperature current in plane magneto resistance measurements on $\mathrm{Py}(300 \AA) / \mathrm{Cr}(41 \AA) / \mathrm{Fe}_{3} \mathrm{O}_{4}(300 \AA)$ spin valves. The shoulders are the antiparallel state. The AMR peak of the Py is unavoidable because of the conductivity mismatch between the metals and the oxide. (b) GMR as a function of Cr spacer layer thickness.

the potential for growing devices to realize magnon-induced spin transfer torques.

\section{Acknowledgments}

The work at USF was supported by the National Science Foundation; the work at UT was separately supported by the National Science Foundation, as well as the Welch Foundation. The authors thank C. Bauer and D. Williams for assistance with some of the measurements.

\section{References}

[1] L. Berger, "Emission of spin waves by a magnetic multilayer traversed by a current," Physical Review B, vol. 54, no. 13, pp. 9353-9358, 1996.

[2] J. C. Slonczewski, "Current-driven excitation of magnetic multilayers," Journal of Magnetism and Magnetic Materials, vol. 159, no. 1-2, pp. L1-L7, 1996.

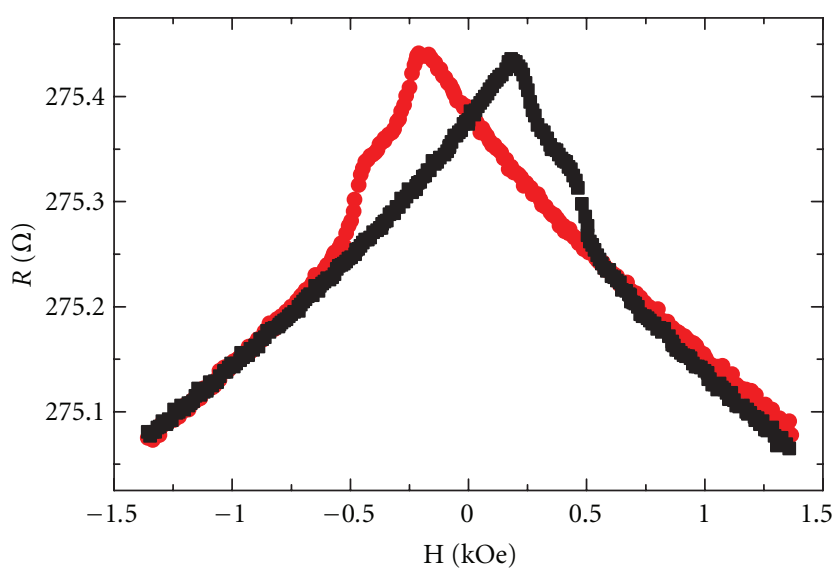

(a)

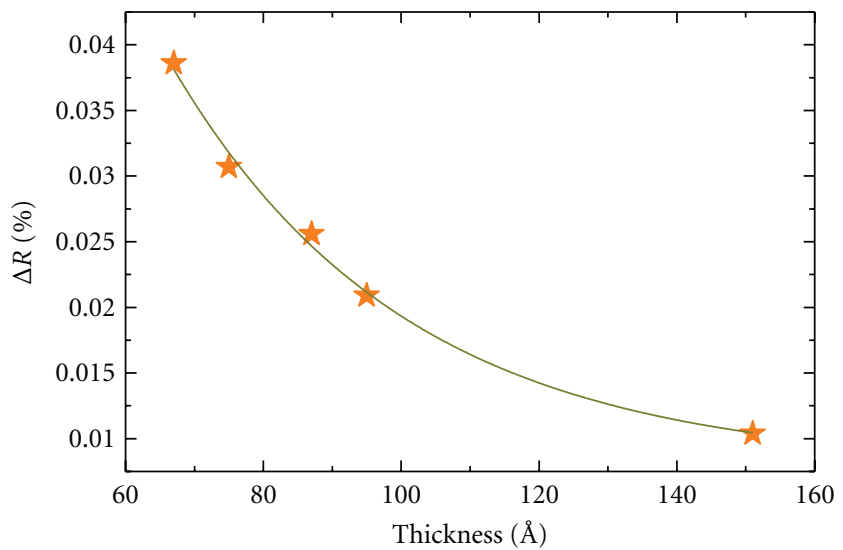

(b)

Figure 7: (a) Current in plane magnetoresistance measurements on $\mathrm{Fe}_{3} \mathrm{O}_{4}(500 \AA) / \mathrm{Cr}(57 \AA) / \mathrm{Fe}_{3} \mathrm{O}_{4}(1000 \AA)$ spin valves. The black (red) data are measurements with increasing (decreasing) fields. (b) Change of GMR as a function of Cr spacer layer thickness; the line is a fit to an exponential decay, with a decay constant of $33 \AA$.

[3] M. Tsoi, A. G. M. Jansen, J. Bass et al., "Excitation of a magnetic multilayer by an electric current," Physical Review Letters, vol. 80, no. 19, pp. 4281-4284, 1998.

[4] E. B. Myers, D. C. Ralph, J. A. Katine, R. N. Louie, and R. A. Buhrman, "Current-induced switching of domains in magnetic multilayer devices," Science, vol. 285, no. 5429, pp. 867-870, 1999.

[5] J. A. Katine, F. J. Albert, R. A. Buhrman, E. B. Myers, and D. C. Ralph, "Current-driven magnetization reversal and spin-wave excitations in Co/Cu/Co pillars," Physical Review Letters, vol. 84, no. 14, pp. 3149-3152, 2000.

[6] J. Åkerman, "Toward a universal memory," Science, vol. 308, no. 5721, pp. 508-510, 2005.

[7] S. I. Klselev, J. C. Sankey, I. N. Krivorotov et al., "Microwave oscillations of a nanomagnet driven by a spin-polarized current," Nature, vol. 425, no. 6956, pp. 380-383, 2003.

[8] I. N. Krivorotov, N. C. Emley, J. C. Sankey, S. I. Kiselev, D. C. Ralph, and R. A. Buhrman, "Time-domain measurements of nanomagnet dynamics driven by spin-transfer torques," Science, vol. 307, no. 5707, pp. 228-231, 2005. 
[9] D. C. Ralph and M. D. Stiles, "Spin transfer torques," Journal of Magnetism and Magnetic Materials, vol. 320, no. 7, pp. 11901216, 2008.

[10] J. C. Slonczewski, "Initiation of spin-transfer torque by thermal transport from magnons," Physical Review B, vol. 82, no. 5, Article ID 054403, 2010.

[11] M. Hatami, G. E. W. Bauer, Q. Zhang, and P. J. Kelly, “Thermal spin-transfer torque in magnetoelectronic devices," Physical Review Letters, vol. 99, no. 6, Article ID 066603, 2007.

[12] J. E. Wegrowe, "Spin transfer from the point of view of the ferromagnetic degrees of freedom," Solid State Communications, vol. 150, no. 11-12, pp. 519-523, 2010.

[13] A. V. Nazarov, H. M. Olson, H. Cho et al., "Spin transfer stimulated microwave emission in $\mathrm{MgO}$ magnetic tunnel junctions," Applied Physics Letters, vol. 88, no. 16, Article ID 162504, 2006.

[14] A. M. Deac, A. Fukushima, H. Kubota et al., "Bias-driven high-power microwave emission from MgO-based tunnel magnetoresistance devices," Nature Physics, vol. 4, no. 10, pp. 803-809, 2008.

[15] D. Houssameddine, S. H. Florez, J. A. Katine et al., "Spin transfer induced coherent microwave emission with large power from nanoscale $\mathrm{MgO}$ tunnel junctions," Applied Physics Letters, vol. 93, no. 2, Article ID 022505, 2008.

[16] M. G. Chapline and S. X. Wang, "Observation of the Verwey transition in thin magnetite films," Journal of Applied Physics, vol. 97, no. 12, Article ID 123901, 3 pages, 2005.

[17] P. B. Jayathilaka, C. A. Bauer, D. V. Williams, M. C. Monti, J. T. Markert, and C. W. Miller, "Impact of ultrathin transition metal buffer layers on $\mathrm{Fe}_{3} \mathrm{O}_{4}$ thin films," Journal of Applied Physics, vol. 107, no. 9, Article ID 09B101, 2010.

[18] A. K. H. Lee, P. B. Jayathilaka, C. A. Bauer et al., "Magnetic force microscopy of epitaxial magnetite films through the Verwey transition," Applied Physics Letters, vol. 97, no. 16, Article ID 162502, 2010.

[19] K. Moloni, B. M. Moskowitz, and E. Dan Dahlberg, "Domain structures in single crystal magnetite below the Verwey transition as observed with a low-temperature magnetic force microscope," Geophysical Research Letters, vol. 23, no. 20, pp. 2851-2854, 1996.

[20] J. Bass and W. P. Pratt Jr., "Spin-diffusion lengths in metals and alloys, and spin-flipping at metal/metal interfaces: an experimentalist's critical review," Journal of Physics Condensed Matter, vol. 19, no. 18, Article ID 183201, 2007.

[21] P. B. Jayathilaka, C. A. Bauer, D. V. Williams, and C. W. Miller, "Influence of growth field on $\mathrm{NiFe}, \mathrm{Fe}_{3} \mathrm{O}_{4}$, and $\mathrm{NiFe} / \mathrm{Cr} / \mathrm{Fe}_{3} \mathrm{O}_{4}$ spin-valves," IEEE Transactions on Magnetics, vol. 46, no. 6, pp. 1777-1779, 2010.

[22] S. van Dijken, X. Fain, S. M. Watts, K. Nakajima, and J. M. D. Coey, "Magnetoresistance of $\mathrm{Fe}_{3} \mathrm{O}_{4} / \mathrm{Au} / \mathrm{Fe}_{3} \mathrm{O}_{4}$ and $\mathrm{Fe}_{3} \mathrm{O}_{4} / \mathrm{Au} / \mathrm{Fe}$ spin-valve structures," Journal of Magnetism and Magnetic Materials, vol. 280, no. 2-3, pp. 322-326, 2004.

[23] M. N. Baibich, J. M. Broto, A. Fert et al., "Giant magnetoresistance of (001)Fe/(001)Cr magnetic superlattices," Physical Review Letters, vol. 61, no. 21, pp. 2472-2475, 1988.

[24] D. Tripathy, A. O. Adeyeye, and S. Shannigrahi, "Effect of spacer layer thickness on the magnetic and magnetotransport properties of $\mathrm{Fe}_{3} \mathrm{O}_{4} / \mathrm{Cu} / \mathrm{Ni}_{80} / \mathrm{Fe}_{20}$ spin valve structures," Physical Review B, vol. 75, no. 1, Article ID 012403, 2007. 

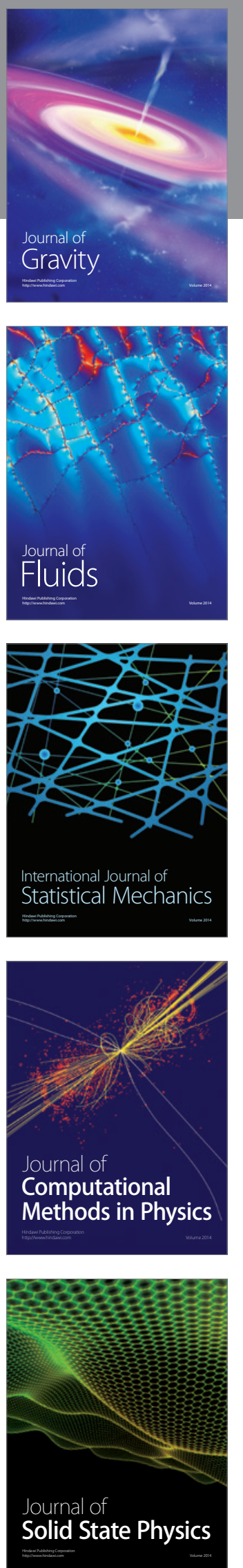

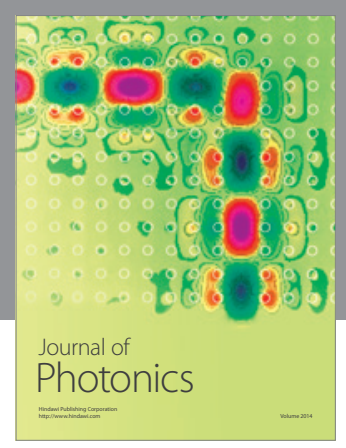

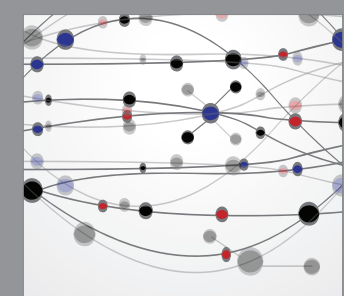

The Scientific World Journal
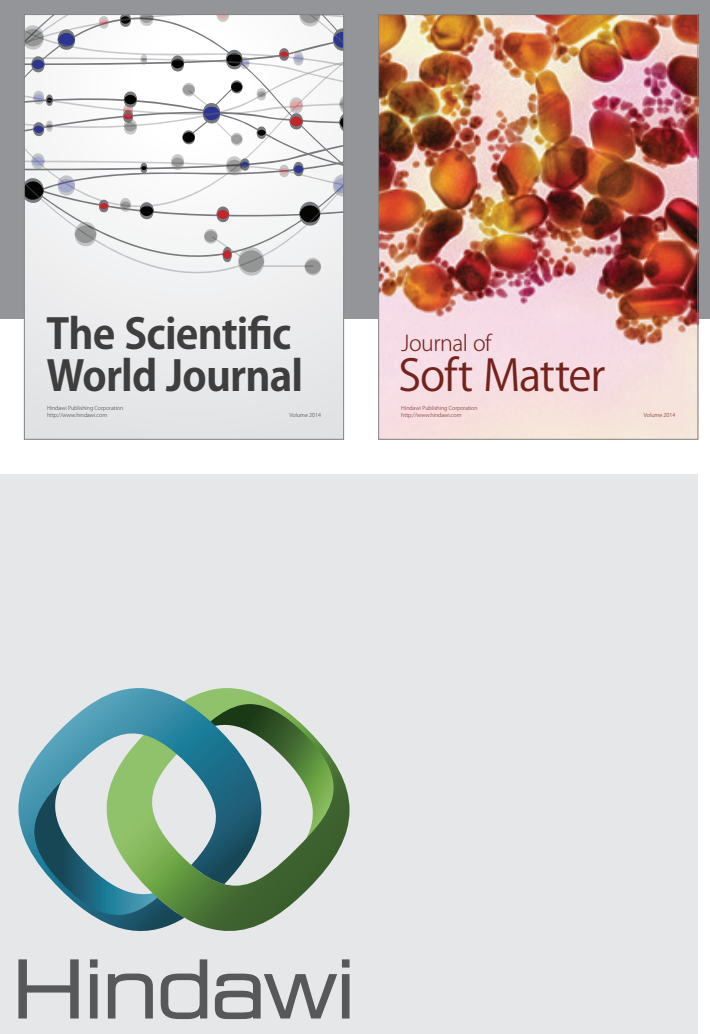

Submit your manuscripts at

http://www.hindawi.com
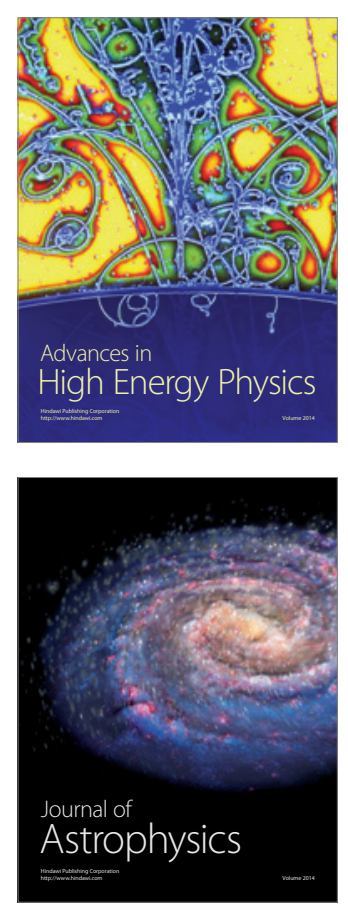
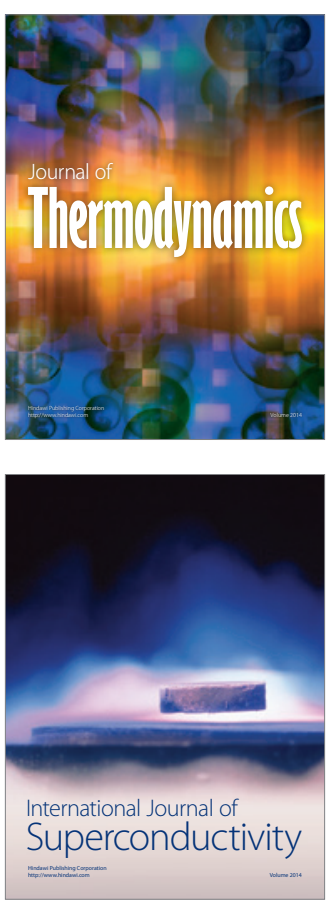
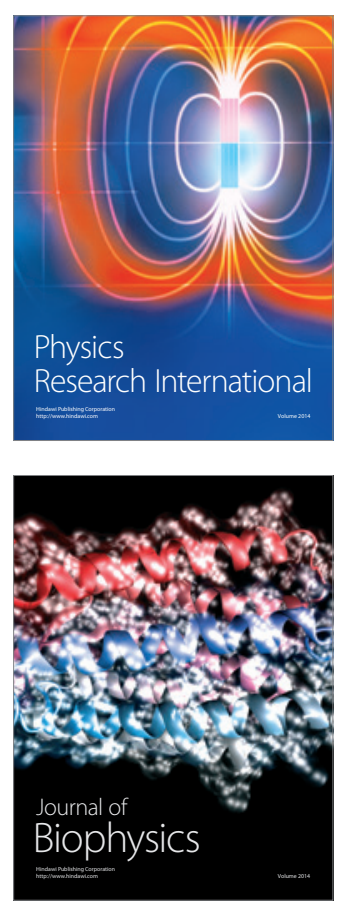
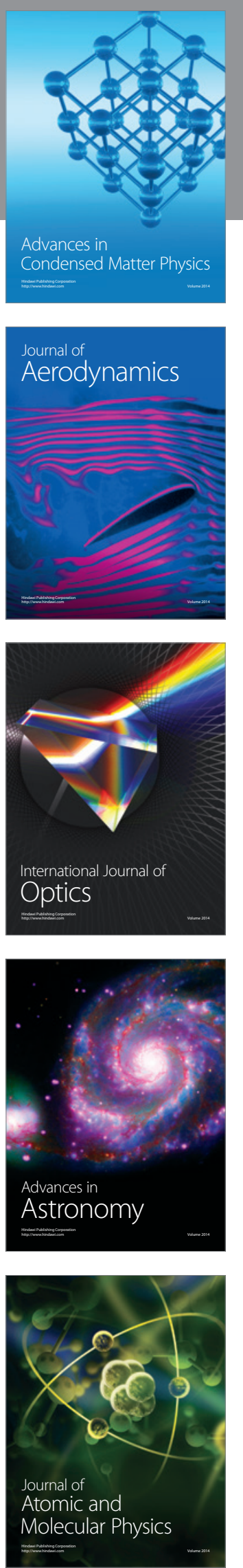